\title{
Aborto, una mirada desde los activismos feministas
}

https://doi.org/10.48102/if.2022.v2.n1.234

\author{
Victoria Raquel Rojas Lozano* \\ vicra.rojas08@gmail.com \\ ORCID: https://orcid.org/0000-0001-6053-9655 \\ Doctora en Ciencias en Salud Colectiva \\ Universidad Autónoma Metropolitana \\ México
}

\author{
María del Rosario Ramírez Morales** \\ mros.rm@gmail.com \\ ORCID: https://orcid.org/0000-0003-4814-6191 \\ Doctora en Ciencias Antropológicas \\ Universidad de Guadalajara \\ México
}

\section{María Belén Herrero Martín***}

beluherrero@hotmail.com

ORCID: https://orcid.org/0000-0002-2850-058X

Maestra en Ciencias Sociales

Universidad Autónoma del Estado de México

México

\begin{abstract}
* Victoria Raquel Rojas Lozano es doctora en Ciencias en Salud Colectiva por la Universidad Autónoma Metropolitana Unidad Xochimilco (UAM-X). Antropóloga feminista, docente en diversas instituciones de educación superior, divulgadora científica, investigadora independiente y directora del Laboratorio Social de Procesos Metodológicos (Labsomet). Madre disidente, abortera autónoma en el Estado de México, acompañante de interrupción legal del embarazo (ILE) en Ciudad de México. Colabora con Balance A. C. y en el programa Fondo de Aborto para la Justicia Social MARÍA; es cofundadora de la Línea de Atención Emocional para Mujeres en Crisis que acciona por la salud mental y emocional de las mujeres.

** María del Rosario Ramírez Morales es doctora en Ciencias Antropológicas por la Universidad Autónoma Metropolitana Unidad Iztapalapa (UAM-I). Profesora investigadora del Departamento de Sociología de la Universidad de Guadalajara (UdeG). Miembro del Sistema Nacional de Investigadores (SNI) y parte del consejo académico de la Red de Investigadores
\end{abstract}

Rojas, V. R., Ramírez, M. del R., Herrero, M. B., Rodríguez, Y. y Rodríguez, M. (2022). Aborto, una mirada desde los activismos feministas. Iberoforum, Revista de Ciencias Sociales, Nueva Época, 2(1), 1-8, Editorial, e000234. https://doi.org/10.48102/if.2022.v2.n1.234

Licencia Pública Internacional - CC BY-NC-ND 4.0 


\author{
Yunitzilim Rodríguez Pedraza**** \\ yunitzilim@outlook.com \\ ORCID: https://orcid.org/0000-0002-7329-2169 \\ Doctora en Derecho \\ Universidad de Quintana Roo \\ México
}

\author{
Margarita Rodríguez Falcón***** \\ ghv1299@hotmail.com \\ ORCID: https://orcid.org/0000-0002-2140-6515 \\ Doctora en Sociología \\ Universidad Juárez Autónoma de Tabasco \\ México
}

del Fenómeno Religioso en México (RIFREM). Consultora en temas de derechos sexuales y reproductivos; colaboradora en proyectos sobre despenalización del aborto y atención a la salud en distintas instancias no gubernamentales a nivel nacional e internacional. Columnista del medio independiente ZonaDocs; autora del libros, artículos y capítulos de libros. Sus investigaciones recientes se han centrado en la intersección entre feminismo, espiritualidad y corporalidad para abordar temas como aborto, menstruación y activismo feminista. *** María Belén Herrero Martín es licenciada en Sociología por la Facultad de Ciencias Sociales de la Universidad Nacional de San Juan (UNSJ) y maestra en Ciencias Sociales con especialidad en Desarrollo Municipal por El Colegio Mexiquense. Actualmente cursa el doctorado en Ciencias Sociales en la UNSJ. Se ha especializado en Ciudad, Seguridad y Participación Ciudadana (diplomado de la Universidad Nacional Autónoma de México y la Universidad Oberta de Cataluña), así como en Género y Ciudad en el Consejo Latinoamericano de Ciencias Sociales (CLACSO). Ha desempeñado su labor docente en distintas instituciones de educación superior; entre ellas, la UNSJ y la Universidad Católica de Cuyo (UCCuyo), ambas en Argentina, así como la Facultad de Ciencias Políticas y Sociales de la Universidad Autónoma del Estado de México (UAEMex), en donde es profesora investigadora de tiempo completo y docente de la especialidad en Género, Violencia y Políticas Públicas. Investigadora con reconocimiento a perfil PRODEP. Actualmente integra el cuerpo académico Género, Espacio y Políticas Públicas en la UAEMex. Sus líneas de investigación son el fenómeno urbano -ciudad, ciudadanía, espacio público, seguridad ciudadana y urbanismo feminista-, género y diversidad sexual y violencia de género.

**** Yunitzilim Rodríguez Pedraza es licenciada en Derecho por la Universidad de Quintana Roo (UQROO); maestra en Derecho Constitucional y Amparo por la Universidad Iberoamericana Golfo-Centro; doctora en Derecho por la Universidad Popular Autónoma de Veracruz (UPAV). Desde 2013 es profesora investigadora de tiempo completo de la UQROO; integrante del cuerpo académico de Estudios Multidisciplinarios en Derechos Humanos y Seguridad Pública. Integrante del grupo de trabajo que se conformó para estudiar la Solicitud de Alerta de Violencia de Género contra las Mujeres (AVGM) en los municipios de Benito Juárez, Cozumel, Isla Mujeres, Lázaro Cárdenas y Solidaridad en el estado de Quintana Roo. Actualmente es integrante del Grupo Interinstitucional y Multidisciplinario (GIM) de seguimiento a la declaratoria de la AVGM. Integrante de la Colectiva Marea Verde Quintana Roo, a través del cual se presentó la iniciativa en el estado para permitir la interrupción legal del embarazo (ILE) hasta la décimo segunda semana y se instó a la Comisión de Derechos

Rojas, V. R., Ramírez, M. del R., Herrero, M. B., Rodríguez, Y. y Rodríguez, M. (2022). Aborto, una mirada desde los activismos feministas. Iberoforum, Revista de Ciencias Sociales, Nueva Época, 2(1), 1-8, Editorial, e000234. https://doi.org/10.48102/if.2022.v2.n1.234 Licencia Pública Internacional - CC BY-NC-ND 4.0 
Aborto, una mirada desde los activismos feministas se publica en un contexto importante para el movimiento feminista mexicano que, después de años de movilización (1930-2021) ${ }^{1}$ y de conseguir la legalización del aborto en la Ciudad de México en 2007, logró la despenalización hasta el primer trimestre de gestación en cinco entidades de la República (Oaxaca, Veracruz, Hidalgo, Baja California y Colima), ${ }^{2}$ cuenta con el precedente histórico de las últimas resoluciones de la Suprema Corte de Justicia de la Nación $(\mathrm{SCJN})^{3} \mathrm{y}$, sobre todo, ha mostrado su efectividad a través del número cada vez mayor de mujeres, niñas y personas gestantes que abortan de forma segura convencidas de que es su derecho. En este sentido, este dossier tiene como objetivo presentar el trabajo realizado por las colectivas de mujeres que han decidido acompañar desde la calle, en la intimidad de la casa, desde el celular o la presencialidad, frente a los Congresos locales o desde el activismo por redes sociales, uno de los procesos de la vida sexual (no) reproductiva: el aborto. La incorporación del tema dentro de la agenda pública y de los espacios de discusión académica se vuelve una tarea cada

Humanos del estado de Quintana Roo (CNDHQROO) a solicitar la ampliación de la AVGM para los municipios de Othón P. Blanco y Felipe Carrillo Puerto por violencia feminicida y por agravio equiparado, misma que ya fue admitida.

$\star \star * * *$ Margarita Rodríguez Falcón es doctora en Sociología por la Benemérita Universidad Autónoma de Puebla (BUAP) y profesora de tiempo completo en la Universidad Juárez Autónoma de Tabasco (UJAT). Cuenta con el perfil PRODEP; es miembro del Sistema Estatal de Investigadores (SEI) y autora de diversas publicaciones.

1 Véase Enríquez (2008); Grupo de Información en Reproducción Elegida [GIRE] (2008, 2018a y 2018b); García y Belausteguigoitia (2012); Lamas (1992, 2005 y 2008).

2 México, aun con la resistencia ejercida por parte de grupos religiosos y políticos conservadores (Ramírez, 2018 y 2021), tiene seis casos exitosos de despenalización del aborto voluntario: el primero ocurrió en la ahora Ciudad de México, cuya propuesta de despenalización del aborto voluntario entró en vigor el 27 de abril de 2007; le siguieron los estados de Oaxaca (28 de octubre de 2019), Hidalgo (30 de junio de 2021), Veracruz (20 de julio de 2021), Baja California (30 de octubre de 2021) y Colima (1 de diciembre de 2021).

3 El 7 de septiembre de 2021, la SCJN resolvió por unanimidad la inconstitucionalidad de la criminalización del aborto y se pronunció por primera vez a favor de garantizar el derecho de las mujeres y personas gestantes a decidir, sin que por ello enfrenten consecuencias penales. Esta decisión histórica sentó precedente para considerar inconstitucionales las normas penales de las entidades federativas que criminalizan el aborto de manera absoluta o las normas que sólo prevén la posibilidad de abortar con excusas absolutorias, pues en esos supuestos la conducta se cataloga como un delito, aunque no se imponga una sanción (Suprema Corte de Justicia de la Nación [SCJN], 2021).

Rojas, V. R., Ramírez, M. del R., Herrero, M. B., Rodríguez, Y. y Rodríguez, M. (2022). Aborto, una mirada desde los activismos feministas. Iberoforum, Revista de Ciencias Sociales, Nueva Época, 2(1), 1-8, Editorial, e000234. https://doi.org/10.48102/if.2022.v2.n1.234 Licencia Pública Internacional - CC BY-NC-ND 4.0 
vez más relevante, tanto para rescatar la perspectiva de las personas directamente involucradas como para destacar el papel de los activismos y la incorporación de la narrativa vivencial de las activistas en la construcción de formas alternativas y encarnadas de nombrar, vivir y defender las decisiones sobre el cuerpo, la sexualidad y la reproducción.

Hasta este momento, pese a los antecedentes ya mencionados, el enfoque del aborto en México sigue una tendencia punitivista y reformista, regulada por los códigos penales de cada entidad federativa. ${ }^{4}$ Es por ello que, ante los vacíos legales y contextos sociales que impiden o dificultan el ejercicio de la interrupción voluntaria del embarazo en contextos idóneos de atención y frente a la sistemática violación a los derechos humanos y la ausencia de servicios integrales de salud sexual y reproductiva, muchas mujeres, niñas y personas con capacidad de gestar han optado por abortos autogestivos, de la mano de otras mujeres y colectivas que acompañan el proceso de interrupción de manera informada y segura, siguiendo estrictos protocolos de atención y mediante una relación horizontal con quienes han decidido transitar por este evento (no) reproductivo.

Actualmente, de manera sistemática en todo el país, existen colectivas y acompañantes autónomas que apoyan de manera voluntaria a las mujeres, niñas y personas con capacidad de gestar que han decidido abortar. Esto debido a que, pese a los avances en la despenalización del aborto en México, sigue siendo una realidad que la interrupción voluntaria del embarazo aún carga con estigma, producto de las ideas conservadoras asociadas con lo religioso y también porque rompe con el estereotipo y mandato de la maternidad obligatoria. Por ello es indispensable crear y entretejer redes de acompañamiento para garantizar la vida y el ejercicio de los derechos sexuales y reproductivos de las mujeres, niñas y personas con capacidad de gestar, en un contexto que culturalmente sigue siendo adverso. De ahí la necesidad de (re)conocer la labor que se realiza desde el activismo social y particularmente el activismo feminista enfocado en la salud sexual y reproductiva.

4 A la fecha, existen ocho excluyentes de responsabilidad legal bajo las cuales se permite el acceso al aborto: violación (32 entidades), imprudencial o culposo (30), peligro de muerte (24), alteraciones graves en el producto (16), salud (16), inseminación artificial no consentida (15), causas económicas (2) y voluntario hasta las doce semanas de gestación (6) (GIRE, 2018b).

Rojas, V. R., Ramírez, M. del R., Herrero, M. B., Rodríguez, Y. y Rodríguez, M. (2022). Aborto, una mirada desde los activismos feministas. Iberoforum, Revista de Ciencias Sociales, Nueva Época, 2(1), 1-8, Editorial, e000234. https://doi.org/10.48102/if.2022.v2.n1.234 Licencia Pública Internacional - CC BY-NC-ND 4.0 
La apuesta por visibilizar una genealogía - apenas incipiente y no terminada- del activismo feminista, mediante la cartografía de su lucha por el aborto y contada a través del puño y letra de sus protagonistas, busca contribuir a la sistematización y recuperación de una historia llena de saberes ancestrales, técnicos y políticos que, al mismo tiempo, nos invita a acompañarlas en el camino que han recorrido para repensar los desafíos y posibilidades de la lucha por el acceso al aborto seguro en México. Esta apuesta también aspira a reconocer la labor que han realizado las redes de activistas y acompañantes a lo largo de la lucha por la despenalización legal y social, y en particular, la impronta de construir otras narrativas que posicionen al aborto como una experiencia de vida, como un derecho al que podemos acceder y que podemos ejercer, así como una cuestión de justicia social y reproductiva. Finalmente, pretende difundir el trabajo y formas de activismo social, político y de cuidados.

Este ejercicio de trabajo colaborativo se posiciona políticamente en favor de construir puentes entre los distintos activismos y la academia. En este sentido, es un exhorto para articular un debate y trabajo abierto y conjunto que debemos emprender en todos los escenarios de nuestro país: desde el Estado, con las universidades y en constante comunicación con la sociedad civil organizada. El diálogo academia-activismo que aquí se enuncia busca escapar a las clásicas etiquetas disciplinares y resistir una lectura encasillada; los y las lectoras encontrarán aquí textos nacidos desde las entrañas de la misma marea verde que las produce y encarna.

La primera sección de este dossier nos muestra algunas de las producciones teóricas feministas, acciones políticas y estrategias legales que se han puesto en marcha en los últimos años, en diferentes estados del país, con respecto al aborto como tema de estudio y de agenda política. Primero, elaborando una crítica al mandato de la maternidad obligatoria y problematizando el cuerpo como territorio a través del concepto de gobernanza reproductiva. En segundo término, apuntando al acompañamiento en el proceso de interrupción del embarazo no deseado, como acción política y estrategia contra el estigma que lo rodea. En tercer lugar, reconociendo el papel del empoderamiento y la capacidad de decidir como elementos esenciales en el ejercicio de los derechos sexuales y reproductivos, mientras se desmitifican las experiencias de incomodidad de las mujeres en el postaborto. Aunado a ello, los últimos textos de esta primera parte muestran la descentralización de la lucha feminista mediante distintas

Rojas, V. R., Ramírez, M. del R., Herrero, M. B., Rodríguez, Y. y Rodríguez, M. (2022). Aborto, una mirada desde los activismos feministas. Iberoforum, Revista de Ciencias Sociales, Nueva Época, 2(1), 1-8, Editorial, e000234. https://doi.org/10.48102/if.2022.v2.n1.234 Licencia Pública Internacional - CC BY-NC-ND 4.0 
formas de manifestación pública, política, simbólica, corporal y legal que se han movilizado a través de las redes y acciones en la defensa del derecho a decidir en Quintana Roo; así también las memorias de un movimiento pionero, como el argentino, desde las especificidades del caso de la provincia de San Juan, el cual ratifica que la lucha se está descentralizando.

En la segunda sección, nuestras colaboradoras dan cuenta, en primera persona, de sus experiencias colectivas en la lucha por la despenalización del aborto, en la búsqueda de justicia reproductiva, así como de los retos, desafíos y acciones encaminados al acompañamiento y el ejercicio del derecho a decidir. Encontramos las reflexiones de las colectivas tabasqueñas y sus experiencias en torno al acompañamiento como fuente de sororidad. Continuamos con el texto de parteras mexicanas que acompañan la salud sexual y reproductiva de las mujeres y que nos exponen los beneficios de la atención por parteras en los servicios de aborto. Cerramos la sección con la experiencia de una colectiva de jóvenes creyentes y feministas que retoman el papel de la religión para el fortalecimiento de las acciones en defensa de la justicia reproductiva de las creyentes que viven procesos de aborto.

La tercera sección nos invita a leer, a partir de sus reseñas, dos de los últimos libros publicados sobre aborto en México: Interrupción del embarazo desde la experiencia de las mujeres: aportaciones interdisciplinarias (2020) y El acceso al aborto en México (2021). Desde la mirada crítica y desde los estudios de género, ambos textos nos muestran al aborto como un evento multidimensional, en donde convergen la política, la salud pública, la educación sexual integral y el derecho. Ambos señalan la necesidad de crear marcos institucionales con una clara y firme perspectiva de género, desde los que se combata el estigma y se reivindiquen elementos íntimos y vivenciales, como la soberanía corporal, las emociones y la autodeterminación.

La cuarta y última sección nos narra, a través de imágenes, el origen del movimiento Marea Verde Quintana Roo, pionero en su género en México, así como las acciones que ha desarrollado, no sólo para el cambio legislativo y político en el estado, sino para la sensibilización social, desmitificación, acompañamiento y capacitación de mujeres respecto al aborto y las violencias de género.

Aborto, una mirada desde los activismos feministas nos interpela para pensar en los problemas que acarrean las políticas punitivistas en torno al

Rojas, V. R., Ramírez, M. del R., Herrero, M. B., Rodríguez, Y. y Rodríguez, M. (2022). Aborto, una mirada desde los activismos feministas. Iberoforum, Revista de Ciencias Sociales, Nueva Época, 2(1), 1-8, Editorial, e000234. https://doi.org/10.48102/if.2022.v2.n1.234 Licencia Pública Internacional - CC BY-NC-ND 4.0 
cuerpo; nos invita a reflexionar sobre la estigmatización que persiste alrededor del aborto; sobre todo, nos invita a (re)conocer otro tipo de activismo. El activismo que se hace desde la intimidad de la compartencia; el que busca que las mujeres, niñas y personas con capacidad de gestar en el corto plazo cambien su vida al decidir sobre su propio cuerpo; el que desde la lucha crea comunidades de cuidado entre desconocidas. El activismo que nos expuso Laura Kaplan en The Story of Jane: The Legendary Underground Feminist Abortion Service (1995); el que nos narró Mabel Bellucci en Historia de una desobediencia. Aborto y feminismo (2014); el que nos contó Raquel Drovetta (2015, 2020 y 2021) a través de la recuperación de la práctica del aborto quirúrgico entre las feministas de los años setenta (en Estados Unidos, Francia e Italia) y los servicios telefónicos que se instalaron en América Latina a inicios del siglo XXI (en Ecuador, Argentina, Chile, Perú y Venezuela). Ese activismo que se vive desde la carne, los afectos y la lucha.

\section{Referencias bibliográficas}

Bellucci, M. (2014). Historia de una desobediencia. Aborto y feminismo. Capital Intelectual.

Drovetta, R. I. (2021). La práctica del aborto en manos de feministas en los 70. Revista Estudos Feministas, 29(2). https://doi.org/10.1590/18069584-2021v29n267176

Drovetta, R. I. (2020). A 50 años del servicio de aborto feminista de Jane en Chicago. Zona Franca, (28), 1-21. https://doi.org/10.35305/zf.vi28.180

Drovetta, R. I. (2015). Líneas telefónicas de información sobre aborto seguro: Una estrategia efectiva para incrementar el acceso de las mujeres a los abortos seguros en América Latina. Reproductive Health Matters, 23(45), 47-57. http://diassere.org.pe/wp-content/ uploads/2019/06/rhm9_12.pdf

Enríquez, L. (2008). Despenalización del aborto en la ciudad de México. Argumentos para la reflexión. Universidad Nacional Autónoma de México, Programa Universitario de Estudios de Género.

Grupo de Información en Reproducción Elegida (2018a). Maternidad o castigo. La criminalización del aborto en México. https://criminalizacionporaborto.gire.org.mx/assets/pdf/Maternidad_o_castigo.pdf

Grupo de Información en Reproducción Elegida (2018b). El aborto en los códigos penales. https://gire.org.mx/plataforma/causales-de-aborto-en-codigos-penales /

Rojas, V. R., Ramírez, M. del R., Herrero, M. B., Rodríguez, Y. y Rodríguez, M. (2022). Aborto, una mirada desde los activismos feministas. Iberoforum, Revista de Ciencias Sociales, Nueva Época, 2(1), 1-8, Editorial, e000234. https://doi.org/10.48102/if.2022.v2.n1.234 Licencia Pública Internacional - CC BY-NC-ND 4.0 
Grupo de Información en Reproducción Elegida (2008). El proceso de despenalización del aborto en la Ciudad de México. https://gire.org.mx/ wp-content/uploads/2016/08/ProcesoDespena_TD7.pdf

García, G. y Belausteguigoitia, M. (eds.) (2012). 20 años por todas las mujeres. GIRE.

Kaplan, L. (1995). The Story of Jane: The Legendary Underground Feminist Abortion Service. University of Chicago Press.

Lamas, M. (2008). El aborto en la agenda del desarrollo en América Latina. Perfiles latinoamericanos, 16(31), 65-93. http://www.scielo.org.mx/ scielo.php?script $=$ sci_arttext\&pid=S0188-76532008000100004

Lamas, M. (2005). Nuevos horizontes de la interrupción legal del embarazo. Desacatos. Revista de Ciencias Sociales, (17), 57-77. https://desacatos. ciesas.edu.mx/index.php/Desacatos/article/view/1057/905

Lamas, M. (1992). El feminismo mexicano y la lucha por legalizar el aborto. Política y cultura, (1), 9-22. https://polcul.xoc.uam.mx/index.php/ polcul/article/view/3/3

Ramírez, M. R. (2021). Entre el verde y el azul: Derechos y antiderechos en la arena pública latinoamericana. En R. de la Torre y P. Semán (eds.), Religiones y espacios públicos en América Latina (pp. 135-154). Centro de Estudios Latinoamericanos Avanzados.

Ramírez, M. R. (2018). Narrativas religiosas y aborto legal. En C. Garma, M. R. Ramírez y A. Corpus (coords.), Familias, Iglesias y Estado laico. Enfoques antropológicos (pp. 423-436). Departamento de Antropología, Universidad Autónoma Metropolitana-Iztapalapa; Ediciones del Lirio.

Suprema Corte de Justicia de la Nación (2021). Comunicados de Prensa. No. 271/2021. Suprema Corte declara inconstitucional la criminalización total del aborto. https://www.internet2.scjn.gob.mx/red2/comunicados/noticia.asp?id=6579

Rojas, V. R., Ramírez, M. del R., Herrero, M. B., Rodríguez, Y. y Rodríguez, M. (2022). Aborto, una mirada desde los activismos feministas. Iberoforum, Revista de Ciencias Sociales, Nueva Época, 2(1), 1-8, Editorial, e000234. https://doi.org/10.48102/if.2022.v2.n1.234 Licencia Pública Internacional - CC BY-NC-ND 4.0 